\title{
DESENVOLVIMENTO DE UM MANIPULADOR ROBÓTICO CONTROLADO POR APLICATIVO UTILIZANDO A METODOLOGIA ABP PARA A DISCIPLINA DE MICROPROCESSADORES
}

Pedro G. Pascoal - pedro.pascoal@ sou.unijui.edu.br

Catherine M. de Freitas - catherine.freitas@ sou.unijui.edu.br

Luís F. Sauthier - luis.sauthier@sou.unijui.edu.br

Douglas F. Copetti - douglas.copetti@ sou.unijui.edu.br

Universidade Regional do Noroeste do Estado do Rio Grande do Sul - UNIJUÍ, Departamento de Ciências Exatas e Engenharias - DCEEng

Rua Lulu Ilgenfritz, Bairro São Geraldo, nº 480.

98700-000 - Ijuí - Rio Grande do Sul

Resumo: O presente trabalho se refere ao desenvolvimento de um manipulador robótico controlado através de potenciômetros ou aplicativo de smartphone via comunicação Bluetooth. $O$ objetivo do projeto foi realizar a aplicação prática dos conhecimentos adquiridos na matéria de microprocessadores através do método de ABP (Aprendizado Baseado em Projeto), onde os estudantes aprimoraram suas habilidades referentes a resolução de problemas tecnológicos reais. Esse modelo de ensino ainda proporcionou a cooperação e envolvimento de todos os alunos, resultando em maior desempenho, bem como em um produto final satisfatório. A disciplina tem ênfase na família de microcontroladores PIC. Para isso, foi projetado um braço robótico em softwares de desenho para montagem mecânica das peças e acoplamento dos motores aos quatro eixos. Após, foi elaborado um código de controle em linguagem $C$ para controlar servomotores através de potenciômetros ou smartphones (comunicações Bluetooth). Esse programa foi implementado no microcontrolador escolhido, o dsPIC33FJ12MC202 da Microchip. Constatou-se que, devido ao modelo de estudos ABP, o empenho e busca por materiais de estudo foi maior por parte dos autores, possibilitando ir além das expectativas no desenvolvimento da planta, uma vez que foi necessário dominar a programação de um microcontrolador específico, bem como os softwares de projeção do manipulador robótico em um espaço de tempo limitado.

Palavras-chave: Aprendizado. Braço robótico. Interdisciplinaridade. Microcontrolador.

\section{INTRODUÇÃO}

A crescente demanda de equipamentos e produtos industrializados têm motivado a busca contínua por produtividade (SANTOS, MANHÃES e LIMA, 2018). De modo que, para a indústria fortalecer e otimizar seu processo de produção, é necessário buscar tendências tecnológicas, aumentando o rendimento e a rentabilidade, possibilitando alternativas de trabalho em ambientes insalubres e não ergonômicos. Dessa forma, há o desenvolvimento de equipamentos capazes de auxiliar o processo de montagem, realizando trabalhos repetitivos e que exijam precisão, de maneira veloz, ininterrupta e padronizada. 
$\mathrm{Na}$ indústria, os equipamentos que podem ser empregados para realizar esses trabalhos são os manipuladores robóticos. Eles são estruturas mecânicas formadas por um conjunto de hastes interligadas por juntas que, de modo geral, comportam motores que possibilitam a mobilidade automática ou controlada. Com a revolução da indústria 4.0, estes mecanismos estão difundidos nas fábricas, exercendo funções variadas que dependem da necessidade que o robô é requisitado. Entre elas podem ser a manipulação, montagem ou corte de equipamentos e peças, independente do ambiente em que são instalados (HUBER; WEISS, 2017).

Este trabalho tem como objetivo desenvolver um manipulador robótico utilizando o método de ABP (Aprendizagem Baseada em Projeto). A motivação surge com o intuito de elaborar um produto aplicando os conteúdos de programação disseminados no decorrer da disciplina de microprocessadores. A técnica ABP consiste em submeter os alunos a desafios para que eles possam traduzir suas experiencias teóricas na resolução de problemas reais. Dessa forma, é necessário compreender o contexto do problema e suas possíveis soluções, utilizando conhecimentos interdisciplinares e materiais disponíveis (BENDER, 2014).

$O$ resultado da aplicação do método de $\mathrm{ABP}$ é um aprendizado mais profundo que integra diversas áreas, onde desenvolve-se habilidades e competências necessárias para o mercado atual. Gary (2015) mostrou em suas pesquisas que, mediante a implementação da metodologia ABP, observa-se que os graduados estão melhor preparados para o trabalho profissional, principalmente em termos de adaptabilidade.

O manipulador robótico desenvolvido neste trabalho possui 4 eixos, divididos entre base, hastes e a garra em sua extremidade. O controle é realizado por um microcontrolador (MCU) da família PIC, o qual é estudado na disciplina de microprocessadores. Os movimentos do braço robótico são baseados em dois modos de operação, sendo eles, a utilização de potenciômetros e o controle via Bluetooth por um dispositivo móvel.

De acordo com a organização do artigo, na seção 2 são apresentados os argumentos para o projeto da estrutura e informações sobre os principais materiais que a compõem. Na seção 3 o software implementado para controle do manipulador e o hardware projetado são descritos. O capítulo 4 compreende o desenvolvimento do aplicativo e, finalmente, na seção 5 são apresentadas as discussões sobre a execução do trabalho e as conclusões dos autores.

\section{PROJETO DA ESTRUTURA}

A estrutura mecânica do manipulador robótico é composta por quatro eixos com 180 graus de liberdade. O primeiro eixo é destinado para a rotação da base. O segundo e o terceiro, realizam o movimento da parte inferior e superior das hastes do braço. Por fim, o quarto eixo é utilizado para fazer a abertura e fechamento da garra. A Figura 1 mostra os eixos de rotação através de uma representação tridimensional do manipulador desenvolvida no software Fusion 360 da Autodesk. As peças bidimensionais (2D) foram projetadas no software AutoCAD, também da Autodesk. O desenvolvimento estrutural foi dividido em cinco partes, conforme a Figura 2. A base fixa do manipulador foi confeccionada com madeira do tipo MDF de $6 \mathrm{~mm}$ de espessura a fim de obter maior resistência e estabilidade mecânica. As demais partes foram feitas de MDF $3 \mathrm{~mm}$ por ser mais leve, contribuindo para a cinemática. As chapas de MDF utilizadas foram cortadas em uma Fresadora CNC. 


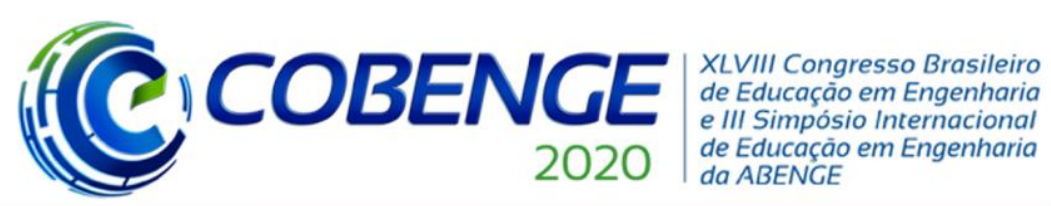

"Os desafios para formar hoje o engenheiro do amanhã"

Figura 1 - Representação tridimensional do manipulador com indicação dos eixos de rotação



Fonte: Autores (2020)

Figura 2 - Peças projetadas no AutoCAD

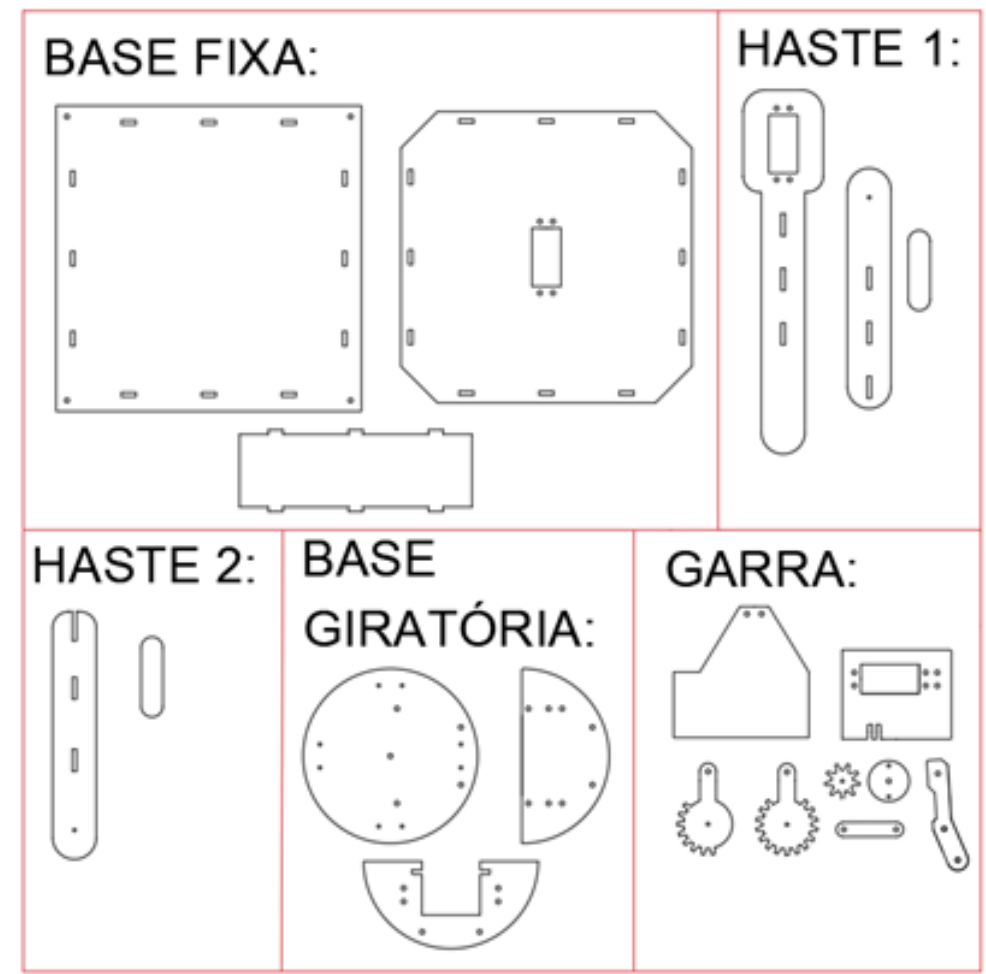

Fonte: Autores (2020)

Os movimentos de rotação foram efetuados por quatro servomotores modelo MG995. Esses motores CC de ímãs permanentes possuem um circuito elétrico interno que possibilita o controle de uma série de movimentos precisos. Dessa forma, através da variação do duty cycle do sinal PWM (Pulse Width Modulation), torna-se possível realizar o controle de posição do motor. O modelo MG995 opera com uma frequência de $50 \mathrm{~Hz}$, onde o duty cycle pode variar entre $0,5 \mathrm{~ms}$ e $2,5 \mathrm{~ms}$, que representam $-90^{\circ}$ e $+90^{\circ}$ respectivamente. Esse motor foi definido através de suas especificações técnicas, que demonstram o fornecimento de torque suficiente 
para realizar os movimentos relativos ao braço robótico, além de possuir engrenagens internas de metal.

O resultado da confecção mecânica está apresentado na Figura 3, onde o manipulador foi submetido à testes, realizando a elevação de um pequeno objeto.

Figura 3 - Estrutura final do braço robótico

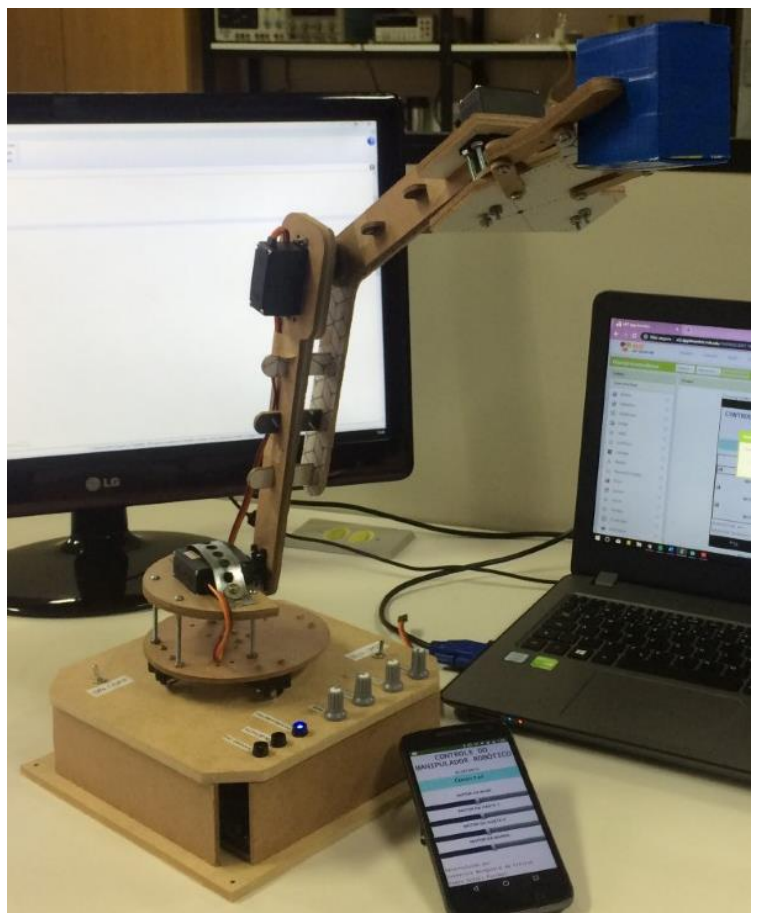

Fonte: Autores (2020)

\section{DESENVOLVIMNTO DE SOFTWARE E HARDWARE}

O controle dos movimentos do manipulador pode ser realizado a partir de dois modos de operação. O primeiro é executado através de potenciômetros e o segundo mediante um aplicativo de celular, utilizando comunicação Bluetooth.

O microcontrolador implementado foi um dsPIC33FJ12MC202 da fabricante Microchip. Ele é um MCU de alto desempenho, com arquitetura Harvard modificada. Possui três timers de 16 bits, onde os timers 2 e 3 podem ser combinados, formando um timer de 32 bits. Esse MCU ainda possui um periférico para controle de motores com oito canais de PWMs com resolução de 16 bits, desses, quatro são definidos por software e outros quatro podem operar no modo complementar ou independente. Ele também dispõe de conversor analógico/digital de 10 ou 12 bits com seis canais de entrada e comunicação SPI, I2C e UART (MICROCHIP TECNOLOGY, 2011).

Esse microcontrolador foi escolhido por apresentar a quantidade necessária de canais de PWM, uma vez que, como comentado anteriormente, o controle de posição dos servomotores é feito através dessa modulação. Dessa forma, foi possível fazer o controle dos quatro motores com maior precisão e simultaneidade, que seria impossível com um microcontrolador da família $16 \mathrm{~F}$, por exemplo. Além disso o MCU contém canais analógicos suficientes para a aplicação, apresentando também, comunicação serial. 




"Os desafios para formar hoje o engenheiro do amanhã"
$\mathrm{O}$ a $\mathrm{O3}$ de dezembro Evento On-line

Para a comunicação Bluetooth foi utilizado o módulo HC-05, ele pode funcionar como mestre ou escravo, operando de $3,3 \mathrm{~V}$ a $5 \mathrm{~V}$, aproximadamente $2,5 \mathrm{GHz}$ e com um alcance de até 10 metros. A comunicação de dados foi executada com o protocolo UART RS232 que realiza a troca de informações binárias em série entre o transmissor e o receptor.

O código foi desenvolvido no compilador CCS em linguagem C. Ele é composto por 3 funções básicas, além da função principal (main). A Figura 4 apresenta, de maneira simplificada, o código desenvolvido para o controle dos motores.

Figura 4 - Código de controle do manipulador em linguagem $\mathrm{C}$

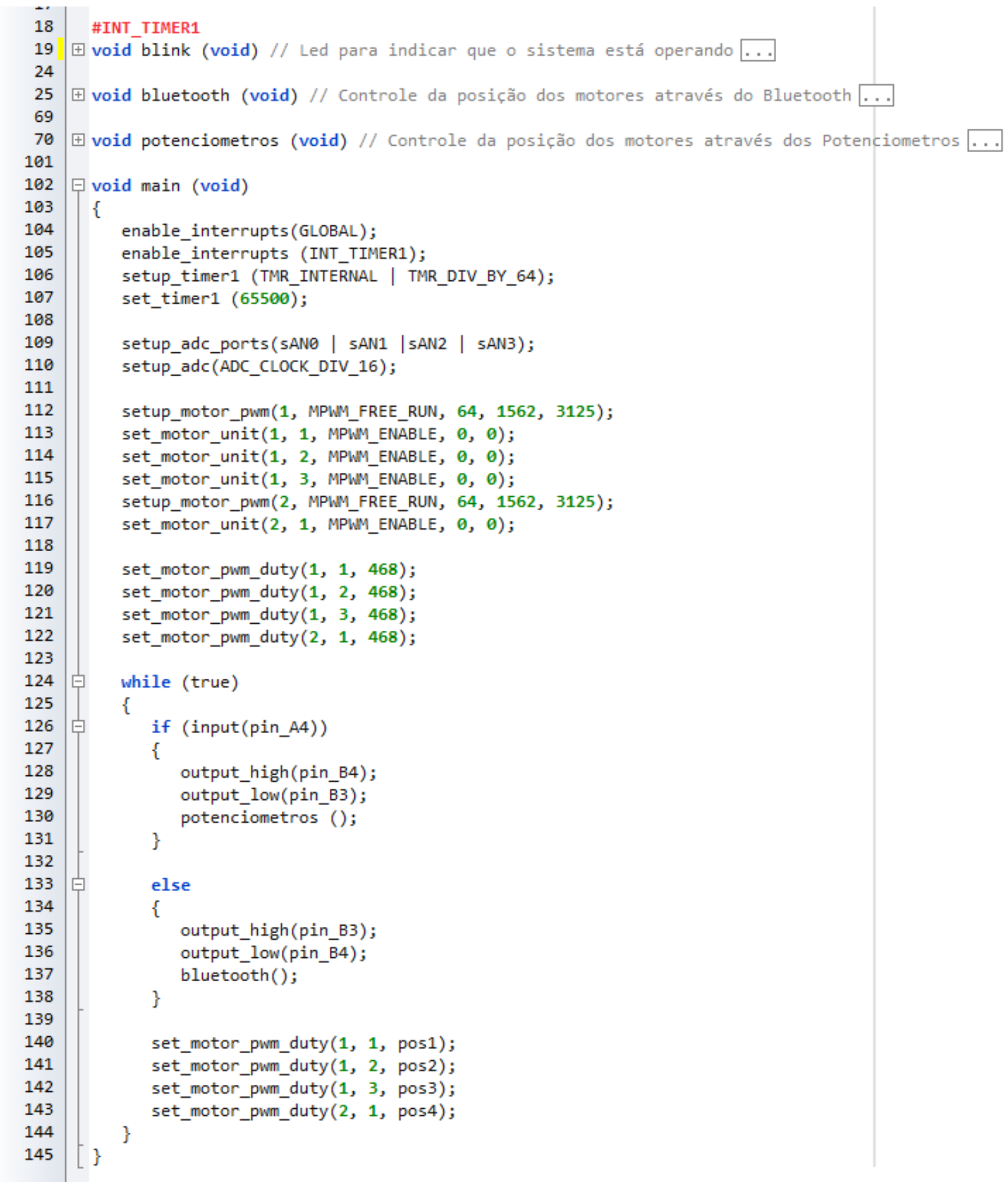

Fonte: Autores (2020) 
(C) COBENGE

"Os desafios para formar hoje o engenheiro do amanhã"
$\mathrm{O}$ a $\mathrm{O3}$ de dezembro Evento On-line

A primeira função (blink) está relacionada ao timer 1 onde é feito o acionamento de um LED para indicar que o sistema está operando corretamente. Na segunda função (bluetooth) é realizado o recebimento dos dados através da porta serial que são refletidos na posição do motor. E na terceira função (potenciometro), ocorre a leitura dos canais analógicos e suas conversões para valores de posição.

Os testes iniciais do código desenvolvido aconteceram com o circuito montado em protoboard. Posterior, foi projetada uma placa de circuito impresso (PCI) utilizando o software Eagle da Autodesk. Nele, foi elaborado o esquemático do projeto e, subsequentemente, a placa, onde ocorreu a disposição dos componentes. A placa pode ser visualizada na Figura 5.

Figura 5 - Placa de Controle do Manipulador

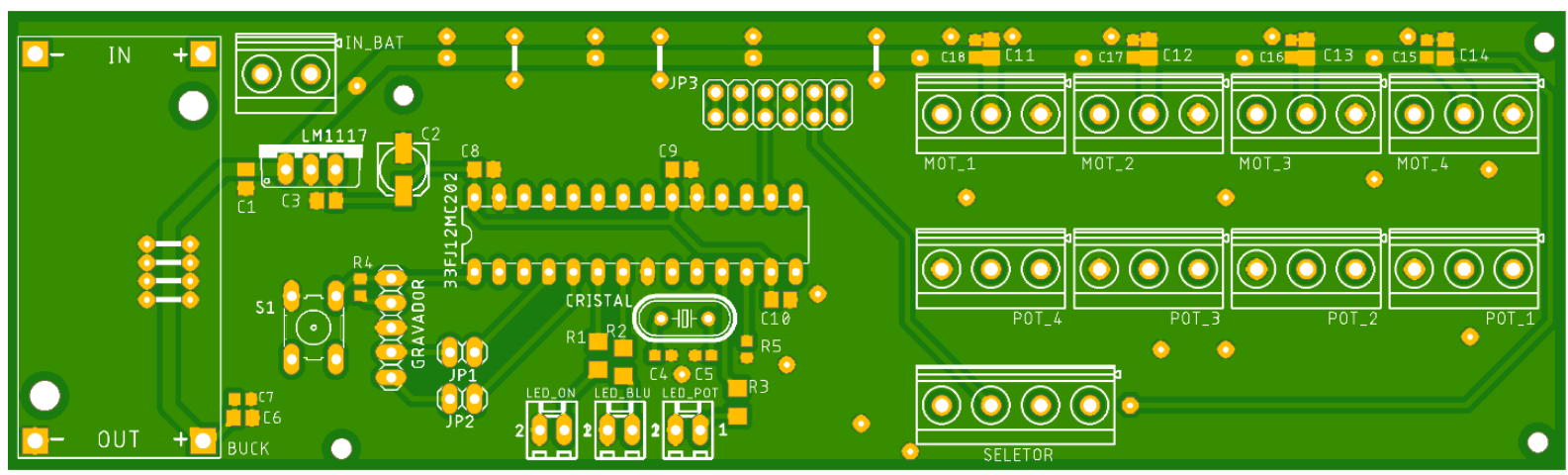

Fonte: Autores (2020)

A partir da Figura 5 é perceptível um borne de 4 vias (seletor), o qual está relacionado a seleção entre o controle do manipulador através dos potenciômetros ou com o smartphone (Bluetooth). A entrada de energia que é aplicada ao conversor Buck, LM2596, é proveniente de um pack de baterias com 6 células de 3,7 V nominal que estão associadas de modo a fornecer uma tensão de saída de 7,4 V. A conexão com a placa ocorre através do borne de duas vias denominado IN_BAT. Dessa forma, o Buck rebaixa a tensão de entrada para 5 V, que é aplicada ao HC-05 e aos servomotores. Para a alimentação do MCU e potenciômetros fez-se necessário o CI LM33V que regula a tensão para 3,3 V. O fluxograma da Figura 6 compreende os níveis de tensão do circuito. 
C COBENCE 2020

"Os desafios para formar hoje o engenheiro do amanhã"

Figura 6 - Fluxograma de níveis de tensão do circuito

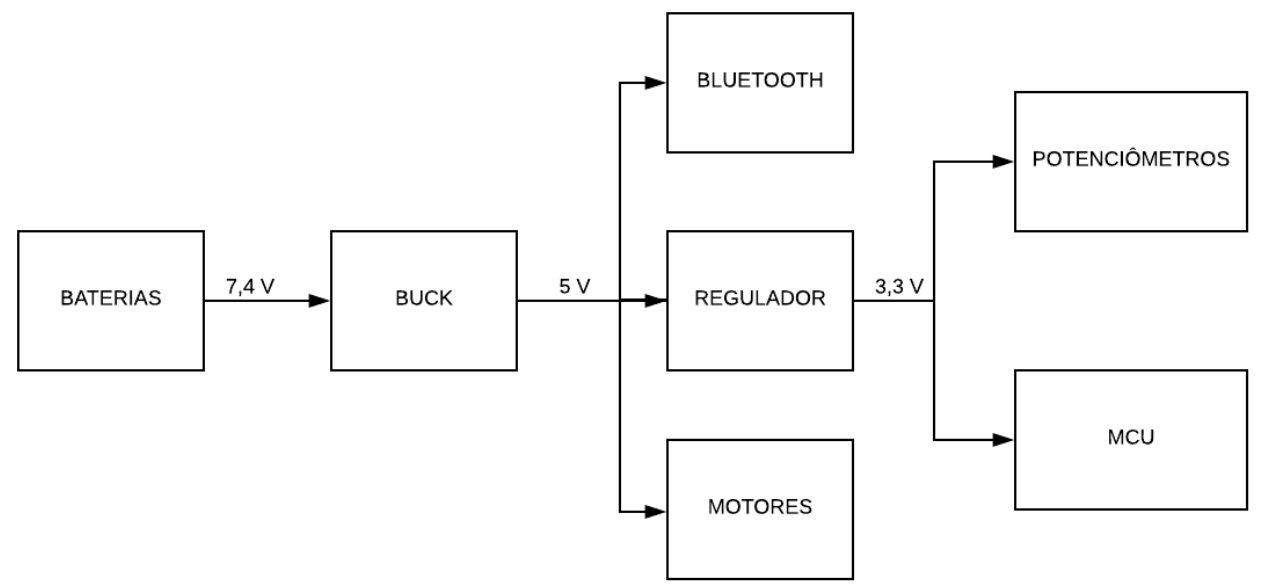

Fonte: Autores (2020)

\section{DESENVOLVIMENTO DO APLICATIVO DE CONTROLE}

O envio dos dados de posição dos motores é realizado através de um smartphone que se conecta ao módulo Bluetooth. O aplicativo para envio de dados foi desenvolvido na plataforma App inventor 2 (AI2) do Massachusetts Institute of Tecnology (MIT).

O AI2 é um ambiente de programação online e gratuito que permite a criação de aplicativos totalmente funcionais para smartphones que contenham o sistema operacional Android. A programação implementada é mais simples e intuitiva, pois é uma linguagem baseada em blocos. O aplicativo também fornece ferramentas gráficas (botões, imagens, textos etc.), contendo opções de utilização de hardwares do próprio dispositivo móvel (Bluetooth, câmera, GPS, acelerômetro etc.). A versão final do aplicativo está mostrada na Figura 7.

Figura 7 - Interface do aplicativo
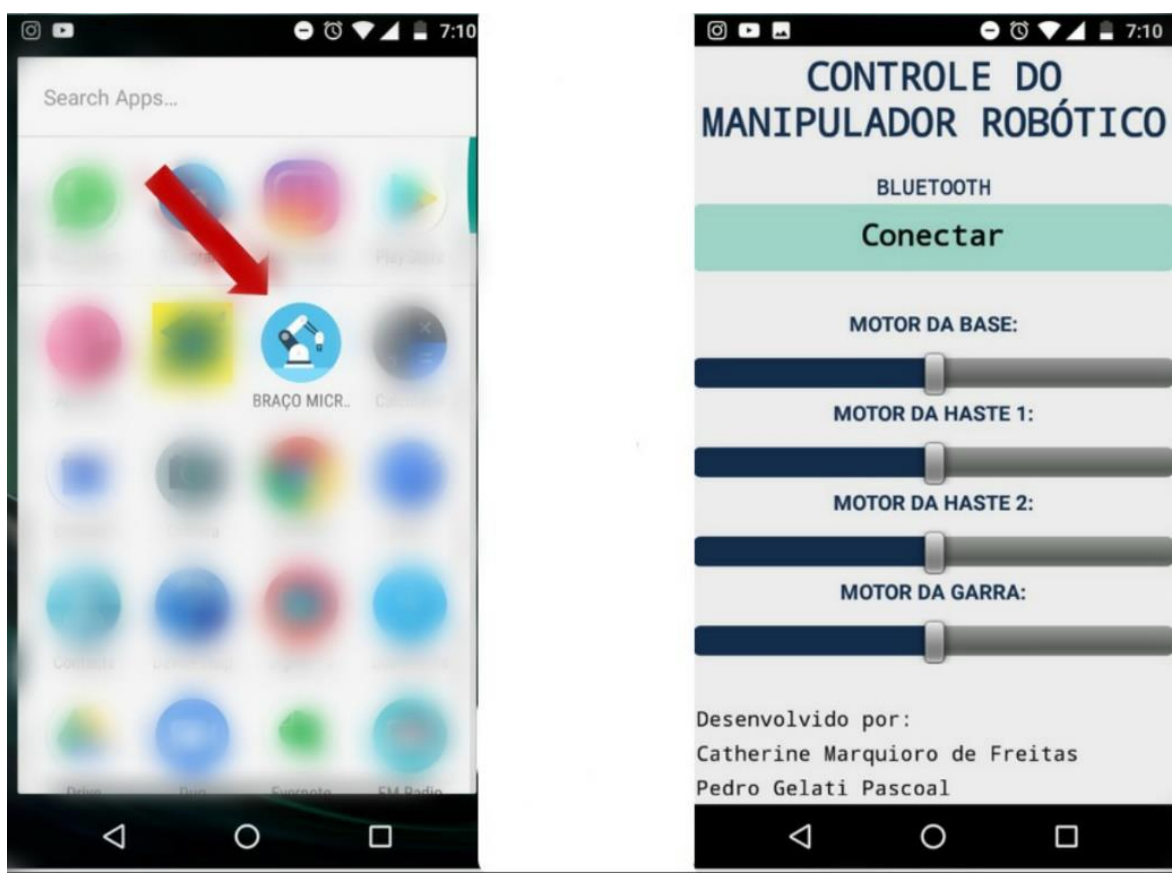

Fonte: Autores (2020) 
A interface do aplicativo é composta por um botão de ativação e conexão do bluetooth, além de 4 sliders que variam a posição para o controle dos motores. Com a movimentação dos sliders o aplicativo faz o envio de 4 bytes, onde o primeiro byte é o cabeçalho, o segundo corresponde ao motor que deve ser acionado $\left(1^{\circ}, 2^{\circ}, 3^{\circ}\right.$ ou $4^{\circ}$ motor). Já o terceiro, representa um valor de 0 a 255 que será refletido na posição do motor. Por fim, o último byte é o rodapé.

\section{CONSIDERAÇÕES FINAIS}

O trabalho tratou da construção de um manipulador robótico de 4 eixos coordenados através do MCU dsPIC33FJ12MC202. O manipulador foi desenvolvido para ser controlado por meio de potenciômetros ou por um dispositivo móvel através de comunicação Bluetooth. As peças do protótipo foram projetadas no AutoCAD e confeccionadas em MDF. O movimento dos eixos foi realizado por quatro servomotores modelo MG995 o qual apresenta o torque necessário para a aplicação do projeto. O código de controle foi desenvolvido no software CCS e a projeção das placas de circuito impresso foram feitas no Eagle. Para o controle via smartphone foi elaborado um aplicativo na plataforma App Inventor 2 do MIT.

$\mathrm{O}$ projeto possibilitou a aplicação da metodologia $\mathrm{ABP}$, a qual oportuniza o desenvolvimento de habilidades como por exemplo o trabalho em equipe, criatividade para a solução de problemas e projeção de estruturas, bem como o reaproveitamento de materiais. Além disso, promoveu vivências práticas e aplicação de um conhecimento híbrido baseado na interdisciplinaridade.

O protótipo ainda foi exposto durante o evento "Profissional do futuro 2019" ministrado pela UNIJUÍ. O evento tem como objetivo despertar curiosidade e interesse de alunos de ensino médio sobre os cursos oferecidos pela instituição. Assim, esses estudantes presenciaram a breve exposição de forma interativa com o braço robótico, sendo introduzidos as possibilidades de atuação do curso de Engenharia Elétrica.

\section{Agradecimentos}

Os autores agradecem a instituição de ensino UNIJUí e ao Grupo de Automação Industrial e Controle (GAIC) pela disponibilidade da infraestrutura e equipamentos necessários para o desenvolvimento do projeto.

\section{REFERÊNCIAS}

BENDER, W. N., Aprendizagem baseada em projetos: Educação diferenciada para o século XXI. Tradução: Fernando de Siqueira Rodrigues. Rio Grande do Sul: Penso Editora, p. 159. 2014.

GARY, K., Project-Based Learning. IEEE Computer Society. Arizona State University. p. 98-100. 2015.

HUBER, A.; WEISS, A. Developing human-robot interaction for an industry 4.0 robot: How industry workers helped to improve remote-hri to physical-hri. In: ACM. Proceedings of the Companion of the 2017 ACM/IEEE International Conference on Human-Robot Interaction. [S.1.], 2017. p. 137-138. 
MICROCHIP TECNOLOGY, dsPIC33FJ12MC201/202 Data Sheet: High-Performance, 16-bit Digital Signal Controllers. p. 304. 2011.

SANTOS, M., MANHÃES, A. M. e LIMA, A. R. Indústria 4.0: desafios e oportunidades para o Brasil. Anais do X Simpósio de Engenharia de Produção de Sergipe. (2018).

\title{
DEVELOPMENT OF A ROBOTIC MANIPULATOR CONTROLLED BY APP USING A PBL METHODOLOGY FOR A MICROPROCESSOR SUBJECT
}

\begin{abstract}
This article refers to development of a robotic manipulator controlled by potentiometers or smartphone app using Bluetooth communication. The objective of the study was to realize the practical application about microprocessor subject through the method of PBL (Project Based Learning), by which students improve their skills referring to resolution of real technological problems. This teaching model also provided the cooperation and involvement of all students, resulting in greater performance, as well as a satisfactory final product. The subject has an emphasis on PIC microcontrollers. For that purpose, a robotic arm was planned and designed in a design software. Afterwards, a code in C language was developed to control servomotors by potentiometers or smartphones (Bluetooth communication). The code was implemented on the Microchip's microcontroller, the dsPIC33FJ12MC202. It was found that, due to the PBL study model, the commitment and search for study materials was greater by authors, making it possible to go beyond expectations in the development of the system, because it was necessary to understand the programming of a specific microcontroller, as well as the robotic manipulator projection software in a limited time.
\end{abstract}

Keywords: Learning. Robotic Arm. Interdisciplinary. Microcontroller. 